\title{
Is there a sex difference in postoperative prognosis of hepatocellular carcinoma?
}

\author{
Ming-Wei Lai ${ }^{1,2,9^{*}} \mathbb{D}$, Yu-De Chu ${ }^{1}$, Chih-Lang Lin ${ }^{1,3}$, Rong-Nan Chien ${ }^{1,3,4}$, Ta-Sen Yeh ${ }^{1,5}$, Tai-Long Pan ${ }^{1,6}$, \\ Po-Yuan Ke $\mathrm{K}^{1,7}$, Kwang-Hui Lin ${ }^{1,8}$ and Chau-Ting Yeh ${ }^{1,4,9^{*}}$
}

\begin{abstract}
Background: Although men carry a higher risk of hepatocellular carcinoma (HCC) than women, it is still controversial whether men also have a poorer postoperative prognosis. A retrospective study was conducted to evaluate the postoperative prognostic predictors of HCC focusing on sex differences.

Methods: We enrolled 516 consecutive adult patients with HCC (118 women, 398 men), who received surgical resection between January 2000 and December 2007, and were followed-up for >10 years. Clinical and laboratory data together with postoperative outcomes were reviewed.

Results: At baseline, female patients had a higher anti-hepatitis $C$ virus antibody prevalence $(P=0.002)$; lower hepatitis $B$ virus surface antigen prevalence $(P=0.006)$; less microvascular invasion $(P=0.019)$; and lower alphafetoprotein $(P=0.023)$, bilirubin $(P=0.002)$, and alanine transaminase $(P=0.001)$ levels. Overall, there were no significant sex differences in terms of intrahepatic recurrence-free survival (RFS), distant metastasis-free survival (MFS), and overall survival (OS). However, subgroup analysis showed that women had favorable RFS $(P=0.019)$ and MFS ( $P=0.034$ ) in patients with alpha-fetoprotein $\leq 35 \mathrm{ng} / \mathrm{mL}$, independent of other clinical variables (adjusted $P=$ 0.008 and 0.043 , respectively). Additionally, men had favorable OS in patients with prothrombin time (international normalized ratio [INR]) $<1.1(P=0.033)$, independent of other clinical variables (adjusted $P=0.042$ ).

Conclusions: Female sex is independently associated with favorable postoperative RFS and MFS in patients with alpha-fetoprotein $\leq 35 \mathrm{ng} / \mathrm{mL}$, while male sex is independently associated with favorable OS in patients with prothrombin time INR $<1.1$.
\end{abstract}

Keywords: Hepatocellular carcinoma, Prognosis, Alpha-fetoprotein, Survival

\section{Background}

Liver cancer is ranked as the sixth most common solid cancer worldwide, with an estimated occurrence of 782,000 new cases each year. It is ranked fifth among cancers in men $(554,000$ cases/year) and ninth among cancers in women $(228,000$ cases/year). Approximately 745,000 people die of liver cancer each year, making it the second leading cause of cancer-related death. It is ranked the second deadliest cancers in men $(521,000$ deaths/year) and the fourth most deadly cancers in women (224,000 deaths/year) [1]. Hepatocellular carcinoma (HCC) is the most common type of primary liver cancer in adults, accounting for approximately $80 \%$ of

\footnotetext{
* Correspondence: a22141@cgmh.org.tw; chautingy@gmail.com

${ }^{1}$ Liver Research Center, Chang Gung Memorial Hospital, Taoyuan, Taiwan Full list of author information is available at the end of the article
}

all liver cancers. Development of HCC is largely associated with chronic hepatitis B virus (HBV) or hepatitis C virus $(\mathrm{HCV})$ infections, as well as environmental toxins including aflatoxin, alcohol, and cigarette smoking $[2,3]$. There is a close geographical correlation between HBV endemic areas and HCC prevalent regions, such as sub-Saharan Africa, China, Hong Kong, and Taiwan [4]. Japan has one of the highest incidence rates of HCV-associated HCC, which appears to be decreasing in recent years, while the incidence in the US has been increasing over the past two decades $[5,6]$.

In almost all parts of the world, men are more likely than women to develop HCC, ranging from 1- (Central America) to 4.8-fold (France) [1, 3, 7]. In the Asia-Pacific region, men are affected 1.3- (Japan) to 4.7-fold (Singapore) more frequently than women [8]. 
The sex disparity in the development of liver cancer is thought to be due to variations in hepatitis carrier states (more hepatitis B infections in men), follow-up/treatment compliance and exposure to environmental toxins $[9,10]$. Androgen/androgen receptor signaling is known to be involved in the initiation of carcinogen-related or HBV-related HCC in men [11], whereas estrogen has been shown to exert protective effects against HCC through interleukin-6 (IL-6) restraints, STAT3 (Signal Transducer and Activator of Transcription-3) inactivation, and tumor-associated macrophage inhibition [12-15].

In Taiwan, which is an HBV endemic region, HBV surface antigen (HBsAg) was found to be positive in around $80 \%$ of male patients with HCC in the 1980s, which gradually decreased to $\sim 70 \%$ by the late 1990 s. A similar trend was also found in women $[16,17]$. A multicenter cohort study enrolling 3483 patients with HCC in Taiwan between 2005 and 2011 showed that the male-to-female ratios were 6:1 in HBV-related, 2:1 in HCV-related, 3:1 in both $\mathrm{HBV} / \mathrm{HCV}$-related and 4:1 in non-B/ non-C-related HCC [18]. The cumulative lifetime incidences of $\mathrm{HCC}$ for men and women, who were positive for $\mathrm{HBsAg}$, were significantly different (27.4\% and $8 \%$, respectively) [19].

Several systems have been proposed to predict the prognosis of HCC, which is more complex than other cancers because of the frequent coexistence of chronic liver disease. The Barcelona Clinic Liver Cancer (BCLC) staging system has shown the optimal independent predictive power of survival when compared with other prognostic systems (Okuda, Tumor-Node-Metastasis (TNM), Cancer of Liver Italian Program (CLIP), Chinese University Prognostic Index (CUPI), Japanese Integrated System (JIS), and Groupe d'Etude de Traitement du Carcinoma Hepatocellulaire (GRETCH)) [20-24]. The survival of HCC is undoubtedly affected by treatment modality, which is applied according to tumor staging $[25,26]$. Notably, none of the prognostic stratification systems have proposed to separate men from women in the evaluation of HCC.

Although sex differences in HCC development risk are well recognized, the prognosis between sexes remains controversial. In a Japanese nationwide survey of 4649 HCC cases, male sex was an independent risk factor for poorer prognosis [24]. Another 12-year single-center series of 704 HCC cases in Japan found a significantly longer survival in women [27]. In an Italian survey of 600 untreated HCC cases, female sex was an independent predictor of better survival [26]. Another Italian study also showed female patients with HCC had longer survival [9]. However, some other series did not demonstrate a sex difference in HCC prognosis [21, 23]. Although female patients with HCC typically present at an older age and with lower tumor burden at diagnosis, female sex was not an independent predictor of survival in an $1886 \mathrm{HCC}$ cases from an American report [28].
Due to the sex disparity in HCC incidence and controversial issues regarding sex differences in HCC prognosis, it is unclear whether postoperative surveillance and management of $\mathrm{HCC}$ require stratification between sexes. To clarify this issue, this retrospective study was conducted to evaluate the postoperative prognostic predictors of HCC that focused on sex differences.

\section{Methods \\ Patients}

The study protocol conformed to the ethical guidelines of the 1975 Declaration of Helsinki as reflected in a priori approval by the Institutional Review Board (201700107B0C501), Chang Gung Memorial Hospital, Taiwan. This retrospective study enrolled 516 consecutive adult patients who were diagnosed with $\mathrm{HCC}$ and received surgical resection at Chang Gung Memorial Hospital between January 2000 and December 2007 and had follow-up durations of up to 10 years. HCC diagnosis was confirmed by the pathologic diagnosis of surgical specimens. In our institute, all HCC patients had to be evaluated before surgery to make sure that a clean margin of $>1 \mathrm{~cm}$ could be achieved. And thus, all our patients had an R0 status. No adjuvant anticancer treatment was given for our patients. Anti-HBV treatment (nucleos $(\mathrm{t})$ ide analogue) was given to chronic hepatitis B patients with serum HBV-DNA levels $>2000 \mathrm{IU} / \mathrm{L}$ according to our National Insurance Policy.

\section{Clinicopathological factors evaluated}

Radiology, operational findings, and pathology reports were reviewed to determine tumor characteristics, including the largest tumor size (the longest diameter), number of tumors, cirrhosis of the non-cancerous liver, histology grade of tumors (grade I to IV based on Edmondson's grading system), branched portal vein invasion (macrovascular invasion), microvascular invasion, capsule, and ascites. Demographic information was retrieved from the charts, including sex, age, HBsAg, anti-HCV antibody, baseline laboratory data (albumin, bilirubin, prothrombin time [PT], international normalized ratio [INR], creatinine, aspartate transaminase [AST], alanine transaminase [ALT], and alpha-fetoprotein [AFP]). Alcoholism was defined as prolonged alcohol abuse leading to psychological and physical dependence.

\section{Statistical analysis}

Continuous data that were normally distributed were reported as the mean \pm standard deviation and categorical variables were expressed as number (\%). Non-parametric data were shown as the median value (range). Comparison of continuous data was performed using the Student's t-test or Mann-Whitney's U test, where appropriate. Comparison of the categorical variables was performed by the Fisher's exact test or Chi-square test with Yates' correction, 
as appropriate. Survival analysis was evaluated by Cox proportional hazard model and verified by Kaplan-Meier analysis. Variables with a P-value $<0.05$ on univariate analysis were included in the multivariate analysis. Statistical comparisons for survival curves were analyzed by the log-rank test. A two-tailed $P$-value $<0.05$ was considered statistically significant.

\section{Results}

\section{Baseline characteristics between male and female patients with HCC}

A total of 516 patients who received surgical resection for HCC were included in this study. Of them, 118 were women and 398 were men. Baseline clinical data are listed in Table 1. The comparison between female and male patients with HCC showed a significant difference in several etiologies: positive anti-HCV was found in 44 $(37.3 \%)$ and $91(22.9 \%)$ patients, respectively $(\mathrm{P}=0.002)$; positive HBsAg was found in $70(59.3 \%)$ and 289 (72.6\%) patients, respectively $(\mathrm{P}=0.006)$; and alcoholism was found in $3(2.5 \%)$ and $130(32.7 \%)$ patients, respectively. More male patients with HCC developed microvascular invasion (men vs. women, 144 [36.2\%] and 29 [24.6\%], P $=0.019$ ). In laboratory data, male patients with HCC had higher AFP levels $(\mathrm{P}=0.023)$; higher bilirubin levels $(P=0.002)$; and higher ALT levels $(P=0.001)$. No significant difference was found for the other parameters.

\section{Comparison between female and male patients for postoperative prognosis including all $\mathbf{5 1 6}$ patients}

Kaplan-Meier analysis was performed to compare postoperative prognosis between female and male patients (Fig. 1). No significant difference was found between the two groups when Log-rank P was calculated (recurrence-free survival: female versus male, mean $(95 \% \mathrm{CI})=59.5$ (48.2 to 70.8 ) versus 53.1 (46.3 to 59.8) months, $\mathrm{P}=0.117$;

Table 1 Comparison between the characteristics of male and female HCCs

\begin{tabular}{|c|c|c|c|}
\hline Clinical variables & Female $(n=118)$ & Male $(n=398)$ & $\mathrm{P}$ \\
\hline Age & $57.7 \pm 14.3$ & $56.2 \pm 13.8$ & 0.310 \\
\hline Anti-HCV positive, n (\%) & $44(37.3 \%)$ & $91(22.9 \%)$ & 0.002 \\
\hline HBsAg positive, n (\%) & $70(59.3 \%)$ & $289(72.6 \%)$ & 0.006 \\
\hline Liver cirrhosis, n (\%) & $67(56.8 \%)$ & $230(57.8 \%)$ & 0.846 \\
\hline Non-cirrhosis, ALT <2XULN a n (\%) & $43(36.4 \%)$ & $125(31.4 \%)$ & 0.361 \\
\hline Non-cirrhosis, ALT > 2xULN, n (\%) & $8(6.8 \%)$ & $43(10.8 \%)$ & 0.267 \\
\hline Microvascular invasion, n (\%) & $29(24.6 \%)$ & $144(36.2 \%)$ & 0.019 \\
\hline Macrovascular invasion, n (\%) & $13(11.0 \%)$ & $54(13.6 \%)$ & 0.469 \\
\hline \multicolumn{4}{|l|}{ Histology grade } \\
\hline$<3$ & $54(45.8 \%)$ & 179 (45.0\%) & \multirow[t]{2}{*}{0.964} \\
\hline$>=3$ & $64(54.2 \%)$ & $219(55.0 \%)$ & \\
\hline Capsule, n (\%) & $86(72.9 \%)$ & $289(72.6 \%)$ & 0.954 \\
\hline \multicolumn{3}{|l|}{ Tumor number } & \multirow[t]{5}{*}{0.817} \\
\hline 1 & $74(62.7 \%)$ & $232(58.3 \%)$ & \\
\hline 2 & $27(22.9 \%)$ & $89(22.4 \%)$ & \\
\hline 3 & $13(11.0 \%)$ & $54(13.6 \%)$ & \\
\hline$>3$ & $4(3.4 \%)$ & $23(5.8 \%)$ & \\
\hline Ascites, n (\%) & $8(6.8 \%)$ & $31(7.8 \%)$ & 0.716 \\
\hline Alcoholism, n (\%) & $3(2.5 \%)$ & $130(32.7 \%)$ & $<0.001$ \\
\hline Largest tumor size, $\mathrm{cm}$ & $5.9 \pm 4.3$ & $6.0 \pm 5.4$ & 0.715 \\
\hline AFP, $n g / m L$ & $25(<1-286980)$ & $58.4(1.1-685353)$ & 0.023 \\
\hline Albumin, g/L & $3.9 \pm 0.6$ & $4.0 \pm 0.6$ & 0.174 \\
\hline Bilirubin, mg/dL & $0.9 \pm 0.6$ & $1.2 \pm 1.4$ & 0.002 \\
\hline Prothrombin time, sec & $11.9 \pm 1.4$ & $12.2 \pm 1.4$ & 0.051 \\
\hline Creatinine, mg/dL & $1.0 \pm 1.1$ & $1.2 \pm 1.0$ & 0.125 \\
\hline AST, U/L & $70.0 \pm 84.8$ & $70.0 \pm 99.0$ & 0.960 \\
\hline$A L T, U / L$ & $53.7 \pm 50.5$ & $79.7 \pm 125.3$ & 0.001 \\
\hline
\end{tabular}

${ }^{a}$ ULN, upper limit of normal; Values in bold, $P<0.05$ 

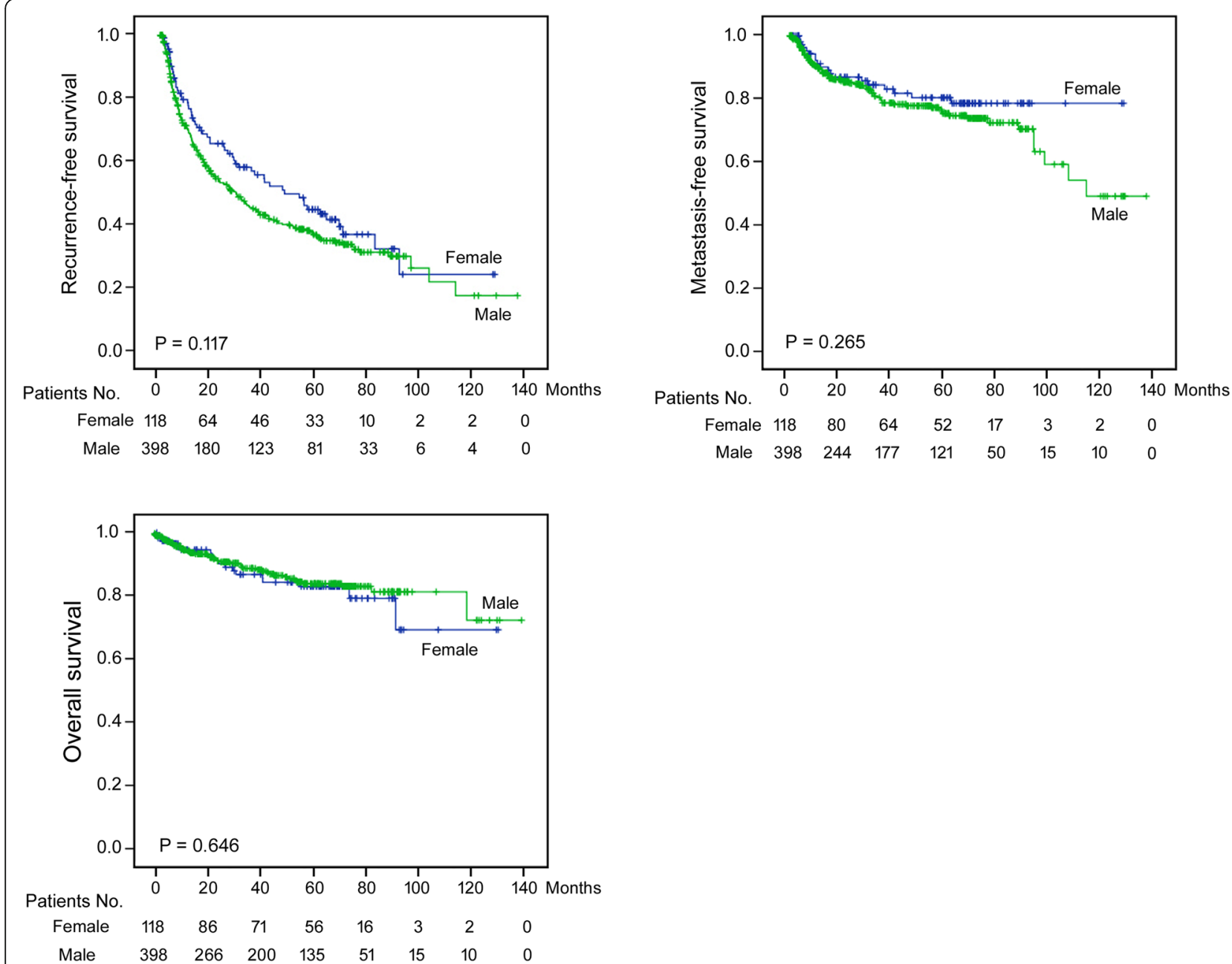

Fig. 1 Survival differences between male and female patients included in this study. All patients were submitted for analysis. Upper left, intrahepatic recurrence-free survivals; Upper right, distant metastasis-free survivals; Lower, overall survivals. Blue curve, female; Green curve, male; The median follow-up period was 43.1 (range 1 to 139) months for all patients

distant metastasis-free survival: 107.3 (98.2 to 116.5$)$ versus 100.0 (92.0 to 107.9 ) months, $\mathrm{P}=0.265$; overall survival: 105.8 (94.8 to 116.8 ) versus 117.2 (111.2 to 123.2$)$ months, $\mathrm{P}=0.646$ ). However, if Breslow (Generalized Wilcoxon) $\mathrm{P}$ was calculated, the $\mathrm{P}$ values were $0.053,0.390$, and 0.826, respectively. Apparently, a borderline (but not significant) P value was found for recurrence-free survival. No patient underwent liver transplantation during the follow-up period. The 1-year, 3-year, and 5-year overall survival rates in male and female patients were about $95 \%$, $90 \%$ and $87 \%$ without significant difference and the details including MFS and RFS were listed in Additional file 1: Table S1

\section{Differential prognosis predictors for male and female patients with HCC}

As an attempt to identify independent risk factors predictive of postoperative survival or recurrence among male and female patients with $\mathrm{HCC}$ and their potential differences, we conducted the analysis separately in male and female patients using the Cox proportional hazard analysis (Additional files 2, 3 and 4: Table S2, S3 and S4). After univariate and multivariate analyses, it was found that male and female patients with HCC had different sets of independent predictors. The significant independent predictors of intrahepatic recurrence-free survival were microvascular invasion $(\mathrm{P}<0.001)$, tumor number $(\mathrm{P}=0.039)$, albumin levels $(\mathrm{P}=0.004)$, and AST levels $(P=0.025)$ for men; for women, the predictors were microvascular invasion $(\mathrm{P}=0.002)$ and AST levels $(\mathrm{P}<0.001)$ (Additional file 2: Table S2). The predictors of metastasis-free survival were microvascular invasion $(P<0.001)$, macrovascular invasion $(P=0.002)$, and AFP levels $(P=0.032)$ for men; for women, the predictors were AFP levels $(\mathrm{P}=0.002)$ and AST levels $(\mathrm{P}=0.001)$ (Additional file 3: Table S3). The predictors 
Table 2 Cox proportional hazard analysis for sex difference in relationship to intrahepatic recurrence-free survival in various clinical subgroups (Male $=1$ )

\begin{tabular}{|c|c|c|c|c|c|}
\hline & & No. of patients & $\mathrm{HR}$ & $95 \% \mathrm{Cl}$ & $\mathrm{P}$ \\
\hline Overall & & 516 & 1.255 & $0.944-1.668$ & 0.118 \\
\hline \multirow[t]{2}{*}{ Age, years } & $<58$ & 262 & 1.448 & $0.912-2.298$ & 0.117 \\
\hline & $\geq 58$ & 254 & 1.162 & $0.801-1.685$ & 0.430 \\
\hline \multirow[t]{2}{*}{ Anti-HCV } & Negative & 381 & 1.329 & $0.928-1.902$ & 0.121 \\
\hline & Positive & 135 & 1.280 & $0.779-2.103$ & 0.330 \\
\hline \multirow[t]{2}{*}{$\mathrm{HBsAg}$} & Negative & 157 & 1.591 & $0.983-2.575$ & 0.059 \\
\hline & Positive & 359 & 1.118 & $0.783-1.598$ & 0.539 \\
\hline \multirow[t]{2}{*}{ Liver cirrhosis } & No & 219 & 1.533 & $0.955-2.460$ & 0.077 \\
\hline & Yes & 297 & 1.080 & $0.754-1.545$ & 0.675 \\
\hline \multirow[t]{2}{*}{ Microvascular invasion } & No & 343 & 1.090 & $0.772-1.539$ & 0.625 \\
\hline & Yes & 173 & 1.203 & $0.710-2.038$ & 0.491 \\
\hline \multirow[t]{2}{*}{ Macrovascular invasion } & No & 449 & 1.283 & $0.943-1.747$ & 0.113 \\
\hline & Yes & 67 & 0.939 & $0.442-1.996$ & 0.870 \\
\hline \multirow[t]{2}{*}{ Histology grade } & $<3$ & 233 & 1.477 & $0.938-2.327$ & 0.092 \\
\hline & $\geq 3$ & 283 & 1.123 & $0.778-1.621$ & 0.534 \\
\hline \multirow[t]{2}{*}{ Capsule } & No & 141 & 1.328 & $0.766-2.303$ & 0.312 \\
\hline & Yes & 375 & 1.219 & $0.873-1.700$ & 0.245 \\
\hline \multirow[t]{2}{*}{ Tumor number } & 1 & 306 & 1.170 & $0.806-1.697$ & 0.409 \\
\hline & $>1$ & 210 & 1.295 & $0.830-2.022$ & 0.255 \\
\hline \multirow[t]{2}{*}{ Ascites } & No & 477 & 1.133 & $0.846-1.516$ & 0.403 \\
\hline & Yes & 39 & 5.798 & $1.336-25.168$ & 0.019 \\
\hline \multirow[t]{2}{*}{ Alcoholism } & No & 383 & 1.227 & $0.905-1.665$ & 0.188 \\
\hline & Yes & 133 & 0.685 & $0.168-2.796$ & 0.598 \\
\hline \multirow[t]{2}{*}{ Largest tumor size, $\mathrm{cm}$} & $\leq 4$ & 242 & 1.176 & $0.784-1.764$ & 0.434 \\
\hline & $>4$ & 274 & 1.276 & $0.853-1.910$ & 0.236 \\
\hline \multirow[t]{2}{*}{ AFP, ng/mL } & $\leq 35$ & 270 & 1.796 & $1.102-2.926$ & 0.019 \\
\hline & $>35$ & 246 & 1.085 & $0.760-1.550$ & 0.653 \\
\hline \multirow[t]{2}{*}{ Albumin, $g / L$} & $\leq 4$ & 279 & 1.372 & $0.937-2.008$ & 0.104 \\
\hline & $>4$ & 237 & 1.139 & $0.741-1.752$ & 0.552 \\
\hline \multirow[t]{2}{*}{ Bilirubin, mg/dL } & $\leq 0.8$ & 263 & 1.336 & $0.913-1.955$ & 0.136 \\
\hline & $>0.8$ & 253 & 1.100 & $0.713-1.696$ & 0.668 \\
\hline \multirow[t]{2}{*}{ PT, sec } & $<12$ & 259 & 1.200 & $0.797-1.808$ & 0.382 \\
\hline & $\geq 12$ & 257 & 1.264 & $0.848-1.885$ & 0.250 \\
\hline \multirow[t]{2}{*}{ PT, INR } & $<1.1$ & 270 & 1.230 & $0.819-1.846$ & 0.319 \\
\hline & $\geq 1.1$ & 246 & 1.261 & $0.844-1.884$ & 0.258 \\
\hline \multirow[t]{2}{*}{ Creatinine, mg/dL } & $\leq 1$ & 294 & 1.256 & $0.906-1.739$ & 0.171 \\
\hline & $>1$ & 222 & 1.342 & $0.625-2.882$ & 0.450 \\
\hline \multirow[t]{2}{*}{ AST, U/L } & $\leq 39$ & 262 & 1.351 & $0.865-2.110$ & 0.186 \\
\hline & $>39$ & 254 & 1.206 & $0.832-1.747$ & 0.323 \\
\hline \multirow[t]{2}{*}{$\mathrm{ALT}, \mathrm{U} / \mathrm{L}$} & $\leq 40$ & 255 & 1.493 & $0.989-2.260$ & 0.058 \\
\hline & $>40$ & 261 & 0.988 & $0.667-1.464$ & 0.954 \\
\hline
\end{tabular}


of overall survival were tumor number $(\mathrm{P}=0.019)$ and albumin levels $(\mathrm{P}=0.045)$ for men, and only bilirubin levels $(P=0.008)$ for women (Additional file 4: Table S4).

\section{Survival and AFP levels}

Subsequently, we performed subgroup analysis to identify subgroups wherein there was a sex difference in term of postoperative prognosis. When intrahepatic recurrence-free survival was compared between all female and male patients with HCC by Cox proportional hazard model, it was found that there was no significant difference (Table 2; $\mathrm{P}=0.118$ ). Subgroup analysis was performed to investigate whether one or more of the subgroups displayed sex difference in terms of recurrence-free survival (Table 2). Median values were used as cut-offs for all continuous variables. It was found that in two subgroups, female patients had a favorable recurrence-free survival: patients with ascites $(\mathrm{n}=39$; $\mathrm{P}$ $=0.019)$ and patients with AFP $\leq 35 \mathrm{ng} / \mathrm{mL}(\mathrm{n}=270 ; \mathrm{P}=$ 0.019). None of the other subgroups showed significant sex differences in terms of recurrence-free survival. When we analyzed the subgroup with AFP $\leq 35 \mathrm{ng} / \mathrm{mL}$ $(\mathrm{n}=270)$, univariate and multivariate analyses both showed sex was an independent predictor of recurrence-free survival (Table 3). When distant metastasis-free survival was compared, it was found that there was no difference between sexes (Table 4; $\mathrm{P}=$ 0.267). However, subgroup analysis showed favorable metastasis-free survival in female patients with lower AFP $\leq 35 \mathrm{ng} / \mathrm{mL}(\mathrm{n}=270 ; \mathrm{P}=0.034)$. None of the other subgroups showed sex differences in terms of distant metastasis-free survival. When we analyzed the subgroup with AFP $\leq 35 \mathrm{ng} / \mathrm{mL}(\mathrm{n}=270)$, sex remained an independent determinant of metastasis-free survival in both univariate and multivariate analyses (Table 5).

\section{Overall survival and PT}

Subsequently, we analyzed sex differences in relation to overall survival in various clinical subgroups. Again, it was found that there was no sex difference, in terms of overall survival, when all patients were compared (Table 6; $\mathrm{P}=0.646$ ). Intriguingly, when subgroup analysis was performed, the subgroup with lower AFP did not show a sex difference $(\mathrm{P}=0.923)$. In contrast, for the subgroups with shorter PT $<12$ sec $(\mathrm{n}=259)$ or INR <1.1 $(\mathrm{n}=270)$, male but not female patients with HCC showed a favorable overall survival $(\mathrm{P}=0.042$ and 0.033 , respectively). Kaplan-Meier analysis also supported this finding (Fig. 2) When we analyzed the subgroup with INR $<1.1$ $(n=270)$, sex remained an independent determinant of overall survival in both univariate and multivariate analyses (Table 7).
Table 3 Cox proportional hazard analysis for clinical variables in relationship to recurrence-free survival in AFP $\leq 35 \mathrm{ng} / \mathrm{mL}$ subgroup $(n=270)$

\begin{tabular}{|c|c|c|c|}
\hline & $\mathrm{HR}$ & $95 \% \mathrm{Cl}$ & $P$ \\
\hline \multicolumn{4}{|l|}{ Univariate analysis } \\
\hline Age, per year & 1.013 & $0.999-1.027$ & 0.070 \\
\hline Gender, Male $=1$ & 1.796 & $1.102-2.926$ & 0.019 \\
\hline Anti-HCV, positive $=1$ & 1.299 & $0.882-1.915$ & 0.186 \\
\hline $\mathrm{HBsAg}$, positive $=1$ & 0.867 & $0.597-1.259$ & 0.454 \\
\hline Liver cirrhosis, yes $=1$ & 1.379 & $0.961-1.979$ & 0.081 \\
\hline Microvascular invasion, yes $=1$ & 2.285 & $1.582-3.302$ & $<0.001$ \\
\hline Macrovascular invasion, yes $=1$ & 2.003 & $1.181-3.398$ & 0.010 \\
\hline Histology grade, per grade & 1.340 & $1.035-1.735$ & 0.026 \\
\hline Capsule, yes $=1$ & 1.040 & $0.709-1.526$ & 0.840 \\
\hline Tumor number, per number & 1.207 & $1.021-1.427$ & 0.027 \\
\hline Ascites yes $=1$ & 2.309 & $1.169-4.561$ & 0.016 \\
\hline Alcoholism yes = 1 & 1.170 & $0.799-1.713$ & 0.419 \\
\hline Largest tumor size, per $\mathrm{cm}$ & 0.997 & $0.970-1.025$ & 0.838 \\
\hline AFP, per $n g / m L$ & 1.034 & $1.014-1.055$ & 0.001 \\
\hline Albumin, per $\mathrm{g} / \mathrm{L}$ & 0.642 & $0.431-0.857$ & 0.003 \\
\hline Bilirubin, per mg/dL & 0.919 & $0.765-1.105$ & 0.371 \\
\hline PT, per sec & 1.074 & $0.966-1.195$ & 0.187 \\
\hline Creatinine, per mg/dL & 1.039 & $0.884-1.220$ & 0.645 \\
\hline AST, per U/L & 1.003 & $1.001-1.004$ & 0.001 \\
\hline$A L T$, per $U / L$ & 1.002 & $1.000-1.003$ & 0.011 \\
\hline \multicolumn{4}{|l|}{ Multivariate analysis } \\
\hline Gender, Male $=1$ & 1.997 & $1.198-3.327$ & 0.008 \\
\hline Microvascular invasion, yes $=1$ & 2.057 & $1.381-3.062$ & $<0.001$ \\
\hline Macrovascular invasion, yes $=1$ & 1.580 & $0.880-2.837$ & 0.126 \\
\hline Histology grade, per grade & 1.075 & $0.816-1.417$ & 0.606 \\
\hline Tumor number, per number & 1.137 & $0.948-1.362$ & 0.166 \\
\hline Ascites yes $=1$ & 1.566 & $0.751-3.265$ & 0.231 \\
\hline AFP, per ng/mL & 1.032 & $1.011-1.054$ & 0.003 \\
\hline Albumin, per $\mathrm{g} / \mathrm{L}$ & 0.709 & $0.521-0.964$ & 0.028 \\
\hline AST, per U/L & 1.002 & $0.998-1.006$ & 0.257 \\
\hline$A L T$, per $U / L$ & 1.000 & $0.997-1.003$ & 0.999 \\
\hline
\end{tabular}

AFP alpha-fetoprotein, $P T$ prothrombin time, AST aspartate aminotransferase, $A L T$ alanine aminotransferase, $H R$ hazard ratio, $\mathrm{Cl}$ confidence interval; Values in bold, $P<0.05$

\section{Discussion}

Women with resectable HCC have different postoperative prognostic predictors from men and have better recurrence-free and metastasis-free survival than men if baseline AFP $<35 \mathrm{ng} / \mathrm{mL}$ from this study. Currently, sex is not considered in diverse prognostic staging systems for HCC. This retrospective, long-term postoperative study intended to clarify this issue. The baseline characteristics showed significantly more $\mathrm{HCV}$ infections, but 
Table 4 Cox proportional hazard analysis for sex difference in relationship to metastasis-free survival in various clinical subgroups $($ Male $=1)$

\begin{tabular}{|c|c|c|c|c|c|}
\hline & & No. of patients & $\mathrm{HR}$ & $95 \% \mathrm{Cl}$ & $P$ \\
\hline Overall & & 516 & 1.328 & $0.805-2.193$ & 0.267 \\
\hline \multirow[t]{2}{*}{ Age, years } & $<58$ & 262 & 0.957 & $0.496-1.847$ & 0.896 \\
\hline & $\geq 58$ & 254 & 1.731 & $0.793-3.779$ & 0.169 \\
\hline \multirow[t]{2}{*}{ Anti-HCV } & Negative & 381 & 1.272 & $0.701-2.307$ & 0.429 \\
\hline & Positive & 135 & 1.369 & $0.523-3.583$ & 0.522 \\
\hline \multirow[t]{2}{*}{$\mathrm{HBsAg}$} & Negative & 157 & 2.159 & $0.876-5.321$ & 0.094 \\
\hline & Positive & 359 & 1.049 & $0.574-1.918$ & 0.877 \\
\hline \multirow[t]{2}{*}{ Liver cirrhosis } & No & 219 & 1.272 & $0.615-2.632$ & 0.517 \\
\hline & Yes & 297 & 1.373 & $0.688-2.742$ & 0.368 \\
\hline \multirow[t]{2}{*}{ Microvascular invasion } & No & 343 & 1.024 & $0.541-1.937$ & 0.943 \\
\hline & Yes & 173 & 1.324 & $0.562-3.119$ & 0.521 \\
\hline \multirow[t]{2}{*}{ Macrovascular invasion } & No & 449 & 1.331 & $0.758-2.340$ & 0.320 \\
\hline & Yes & 67 & 1.147 & $0.379-3.477$ & 0.808 \\
\hline \multirow[t]{2}{*}{ Histology grade } & $<3$ & 233 & 2.094 & $0.816-5.370$ & 0.124 \\
\hline & $\geq 3$ & 283 & 1.076 & $0.592-1.958$ & 0.809 \\
\hline \multirow[t]{2}{*}{ Capsule } & No & 141 & 0.868 & $0.366-2.056$ & 0.747 \\
\hline & Yes & 375 & 1.550 & $0.831-2.890$ & 0.168 \\
\hline \multirow[t]{2}{*}{ Tumor number } & 1 & 306 & 1.242 & $0.639-2.415$ & 0.523 \\
\hline & $>1$ & 210 & 1.362 & $0.631-2.940$ & 0.431 \\
\hline \multirow[t]{2}{*}{ Ascites } & No & 477 & 1.246 & $0.753-2.061$ & 0.392 \\
\hline & Yes & 39 & 31.863 & $0.002-458965$ & 0.479 \\
\hline \multirow[t]{2}{*}{ Alcoholism } & No & 383 & 1.248 & $0.736-2.116$ & 0.411 \\
\hline & Yes & 133 & 20.884 & $0.000-1274875$ & 0.589 \\
\hline \multirow[t]{2}{*}{ Largest tumor size, $\mathrm{cm}$} & $\leq 4$ & 242 & 1.851 & $0.769-4.456$ & 0.169 \\
\hline & $>4$ & 274 & 1.060 & $0.575-1.952$ & 0.852 \\
\hline \multirow[t]{2}{*}{ AFP, ng/mL } & $\leq 35$ & 270 & 3.572 & $1.104-11.553$ & 0.034 \\
\hline & $>35$ & 246 & 0.956 & $0.534-1.714$ & 0.881 \\
\hline \multirow[t]{2}{*}{ Albumin, $g / L$} & $\leq 4$ & 279 & 1.378 & $0.728-2.606$ & 0.325 \\
\hline & $>4$ & 237 & 1.356 & $0.601-3.061$ & 0.463 \\
\hline \multirow[t]{2}{*}{ Bilirubin, mg/dL } & $\leq 0.8$ & 263 & 1.418 & $0.746-2.694$ & 0.287 \\
\hline & $>0.8$ & 253 & 1.154 & $0.511-2.604$ & 0.730 \\
\hline \multirow[t]{2}{*}{ PT, sec } & $<12$ & 259 & 1.105 & $0.559-2.184$ & 0.774 \\
\hline & $\geq 12$ & 257 & 1.572 & $0.740-3.340$ & 0.239 \\
\hline \multirow[t]{2}{*}{ PT, INR } & $<1.1$ & 270 & 1.189 & $0.607-2.328$ & 0.614 \\
\hline & $\geq 1.1$ & 246 & 1.496 & $0.700-3.195$ & 0.299 \\
\hline \multirow[t]{2}{*}{ Creatinine, mg/dL } & $\leq 1$ & 294 & 1.325 & $0.776-2.265$ & 0.303 \\
\hline & $>1$ & 222 & 22.955 & $0.098-5387$ & 0.261 \\
\hline \multirow[t]{2}{*}{ AST, U/L } & $\leq 39$ & 262 & 1.124 & $0.538-2.351$ & 0.756 \\
\hline & $>39$ & 254 & 1.511 & $0.761-3.000$ & 0.238 \\
\hline \multirow[t]{2}{*}{$\mathrm{ALT}, \mathrm{U} / \mathrm{L}$} & $\leq 40$ & 255 & 1.695 & $0.849-3.384$ & 0.134 \\
\hline & $>40$ & 261 & 1.040 & $0.503-2.153$ & 0.915 \\
\hline
\end{tabular}


Table 5 Cox proportional hazard analysis for clinical variables in relationship to metastasis-free survival in AFP $\leq 35 \mathrm{ng} / \mathrm{mL}$ subgroup $(n=270)$

\begin{tabular}{|c|c|c|c|}
\hline & $H R$ & $95 \% \mathrm{Cl}$ & $P$ \\
\hline \multicolumn{4}{|l|}{ Univariate analysis } \\
\hline Age, per year & 1.017 & $0.992-1.042$ & 0.188 \\
\hline Gender, Male $=1$ & 3.572 & $1.104-11.553$ & 0.034 \\
\hline Anti-HCV, positive $=1$ & 1.208 & $0.618-2.360$ & 0.581 \\
\hline HBsAg, positive $=1$ & 0.866 & $0.456-1.643$ & 0.660 \\
\hline Liver cirrhosis, yes $=1$ & 1.125 & $0.610-2.074$ & 0.707 \\
\hline Microvascular invasion, yes $=1$ & 2.742 & $1.491-5.041$ & 0.001 \\
\hline Macrovascular invasion, yes $=1$ & 4.081 & $1.999-8.332$ & $<0.001$ \\
\hline Histology grade, per grade & 1.473 & $0.951-2.281$ & 0.083 \\
\hline Capsule, yes $=1$ & 0.980 & $0.511-1.880$ & 0.952 \\
\hline Tumor number, per number & 1.308 & $1.007-1.700$ & 0.044 \\
\hline Ascites yes $=1$ & 1.048 & $0.253-4.348$ & 0.948 \\
\hline Alcoholism yes = 1 & 1.043 & $0.535-2.033$ & 0.9903 \\
\hline Largest tumor size, per $\mathrm{cm}$ & 1.009 & $0.973-1.047$ & 0.613 \\
\hline AFP, per ng/mL & 1.037 & $1.003-1.073$ & 0.035 \\
\hline Albumin, per $\mathrm{g} / \mathrm{L}$ & 0.740 & $0.442-1.239$ & 0.252 \\
\hline Bilirubin, per mg/dL & 0.954 & $0.713-1.275$ & 0.748 \\
\hline PT, per sec & 1.084 & $0.903-1.302$ & 0.385 \\
\hline Creatinine, per mg/dL & 0.891 & $0.567-1.399$ & 0.615 \\
\hline AST, per U/L & 1.001 & $0.998-1.004$ & 0.534 \\
\hline$A L T$, per $U / L$ & 1.000 & $0.996-1.003$ & 0.787 \\
\hline \multicolumn{4}{|l|}{ Multivariate analysis } \\
\hline Gender, Male $=1$ & 3.413 & $1.042-11.183$ & 0.043 \\
\hline Microvascular invasion, yes $=1$ & 2.687 & $1.426-5.060$ & 0.002 \\
\hline Macrovascular invasion, yes $=1$ & 4.209 & $1.867-9.490$ & 0.001 \\
\hline Tumor number, per number & 1.004 & $0.749-1.347$ & 0.977 \\
\hline AFP, per $n g / m L$ & 1.052 & $1.015-1.089$ & 0.005 \\
\hline
\end{tabular}

AFP alpha-fetoprotein, PT prothrombin time, AST aspartate aminotransferase, $A L T$ alanine aminotransferase, $H R$ hazard ratio, $\mathrm{Cl}$ confidence interval; Values in bold, $P<0.05$

less HBV infections and alcoholism in women with $\mathrm{HCC}$, which is the same etiologic spectrum as previous literature, especially in Asian populations, except the Japanese $[9,28]$. Higher baseline AFP, ALT, and bilirubin levels, as well as microvascular invasion, were also characteristics of men with $\mathrm{HCC}$, which indicates more aggressive tumor behavior, background hepatic necroinflammation, and poor liver reserves; this would be expected to affect long-term, post-operative prognoses. However, there was no significant difference in intrahepatic recurrence-free survival or distant metastasis-free survival when all female and male patients were compared. After stratification for various clinical parameters, female patients with HCC showed better recurrence-free and metastasis-free survival in those with AFP $\leq 35 \mathrm{ng} / \mathrm{mL}$ or those with ascites. AFP is an important biomarker in predicting HCC outcome and is incorporated into several staging systems. However, the recommended cut-off levels of AFP vary. In CLIP, the cut-off level is $400 \mathrm{ng} / \mathrm{mL}$ (score 0, 1); in GRETCH, $35 \mathrm{ng} / \mathrm{mL}$ (score 0, 2); and in CUPI, $500 \mathrm{ng} / \mathrm{mL}$ (score 2) [29-31]. An AFP staging system sets levels of 10-150, 150-500, and $>500 \mathrm{ng} / \mathrm{mL}$ for the discrimination of survival, especially in non-cirrhotic cases [32]. Lower AFP levels indicate either more favorable tumor characteristics (microvascular invasion, differentiation), less tumor burden, or non-cirrhotic background, which all imply an early HCC stage. At an early stage of $\mathrm{HCC}$, female sex hormones may exert a protective role, whereas androgen may exert initiation/ promotion effects on the tumor during this phase. In patients with higher AFP, the growth regulatory effects from other signaling pathways, such as tyrosine kinase receptor-related pathways, might play a more important role that masks the effects from sex hormones.

Ascites indicates either poor liver reserves, portal hypertension, or portal vein thrombosis, which are all predictors of poor prognosis and is represented as an individual factor (Okuda, CUPI, Advanced Liver Cancer Prognostic System) or Child-Turcotte-Pugh scores in several staging systems (CLIP, BCLC, JIS, etc.) [33, 34]. However, in this operable cohort, only 39 patients presented with ascites. Better prognosis in women than men needs further validation.

Regarding overall survival, the sex analysis paradoxically favors male patients with HCC in the subgroup with good coagulation profiles (PT or INR). The reason for this seemingly contradictory observation is unclear. A possible explanation is that men, usually physically stronger, may withstand repetitive therapies, such as transarterial chemoembolization (TACE) for recurrent tumors over a long duration. Alternatively, androgen has dual but opposite effects on hepatocarcinogenesis: initiation and promotion at an early stage, whereas suppression of metastasis at a late stage, which may explain the longer overall survival in men with good liver reserves [35].

When the clinical features were separately analyzed in male and female patients with HCC, it is intriguing to discover that for different endpoints (recurrence-free survival, metastasis-free survival or overall survival), different or additional clinical features accounted for HCC outcome in male patients. This reflects the fact that sex itself may exert certain biological effects on the natural course of HCC.

HCC is a sexual dimorphic cancer with male predilection, not only in humans but also in rodents. Sex hormones are expected to play a central role in the sexual disparity of this malignancy. Li et al. found that androgen/ androgen receptor (AR) signaling mediated 
Table 6 Cox proportional hazard analysis for sex difference in relationship to overall survival in various clinical subgroups (Male $=1$ )

\begin{tabular}{|c|c|c|c|c|c|}
\hline & & No. of patients & $\mathrm{HR}$ & $95 \% \mathrm{Cl}$ & $P$ \\
\hline Overall & & 516 & 0.881 & $0.513-1.513$ & 0.646 \\
\hline \multirow[t]{2}{*}{ Age, years } & $<58$ & 262 & 1.671 & $0.590-4.735$ & 0.334 \\
\hline & $\geq 58$ & 254 & 0.567 & $0.279-1.152$ & 0.117 \\
\hline \multirow[t]{2}{*}{ Anti-HCV } & Negative & 381 & 0.892 & $0.468-1.701$ & 0.729 \\
\hline & Positive & 135 & 0.813 & $0.288-2.291$ & 0.695 \\
\hline \multirow[t]{2}{*}{$\mathrm{HBsAg}$} & Negative & 157 & 0.556 & $0.236-1.309$ & 0.179 \\
\hline & Positive & 359 & 1.244 & $0.580-2.669$ & 0.575 \\
\hline \multirow[t]{2}{*}{ Liver cirrhosis } & No & 219 & 1.573 & $0.602-4.111$ & 0.356 \\
\hline & Yes & 297 & 0.625 & $0.318-1.228$ & 0.172 \\
\hline \multirow[t]{2}{*}{ Microvascular invasion } & No & 343 & 0.830 & $0.420-1.641$ & 0.593 \\
\hline & Yes & 173 & 0.751 & $0.304-1.854$ & 0.535 \\
\hline \multirow[t]{2}{*}{ Macrovascular invasion } & No & 449 & 0.924 & $0.502-1.699$ & 0.798 \\
\hline & Yes & 67 & 0.629 & $0.189-2.102$ & 0.452 \\
\hline \multirow[t]{2}{*}{ Histology grade } & $<3$ & 233 & 1.005 & $0.403-2.509$ & 0.991 \\
\hline & $\geq 3$ & 283 & 0.831 & $0.424-1.630$ & 0.590 \\
\hline \multirow[t]{2}{*}{ Capsule } & No & 141 & 0.621 & $0.231-1.617$ & 0.322 \\
\hline & Yes & 375 & 0.987 & $0.512-1.903$ & 0.970 \\
\hline \multirow[t]{2}{*}{ Tumor number } & 1 & 306 & 0.671 & $0.344-1.306$ & 0.240 \\
\hline & $>1$ & 210 & 1.349 & $0.512-3.555$ & 0.545 \\
\hline \multirow[t]{2}{*}{ Ascites } & No & 477 & 0.880 & $0.487-1.591$ & 0.672 \\
\hline & Yes & 39 & 0.965 & $0.254-3.662$ & 0.958 \\
\hline \multirow[t]{2}{*}{ Alcoholism } & No & 383 & 0.768 & $0.426-1.385$ & 0.381 \\
\hline & Yes & 133 & 20.814 & $0.000-29575628$ & 0.675 \\
\hline \multirow[t]{2}{*}{ Largest tumor size, cm } & $\leq 4$ & 242 & 0.609 & $0.252-1.470$ & 0.270 \\
\hline & $>4$ & 274 & 1.023 & $0.507-2.062$ & 0.950 \\
\hline \multirow[t]{2}{*}{ AFP, ng/mL } & $\leq 35$ & 270 & 1.003 & $0.374-2.689$ & 0.996 \\
\hline & $>35$ & 246 & 0.968 & $0.504-1.858$ & 0.923 \\
\hline \multirow[t]{2}{*}{ Albumin, $g / L$} & $\leq 4$ & 279 & 1.064 & $0.519-2.180$ & 0.866 \\
\hline & $>4$ & 237 & 0.658 & $0.288-1.504$ & 0.321 \\
\hline \multirow[t]{2}{*}{ Bilirubin, mg/dL } & $\leq 0.8$ & 263 & 0.674 & $0.327-1.390$ & 0.285 \\
\hline & $>0.8$ & 253 & 1.129 & $0.469-2.721$ & 0.786 \\
\hline \multirow[t]{2}{*}{ PT, sec } & $<12$ & 259 & 0.455 & $0.213-0.972$ & 0.042 \\
\hline & $\geq 12$ & 257 & 1.61 & $0.671-3.819$ & 0.289 \\
\hline \multirow[t]{2}{*}{ PT, INR } & $<1.1$ & 270 & 0.438 & $0.205-0.936$ & 0.033 \\
\hline & $\geq 1.1$ & 246 & 1.677 & $0.703-4.001$ & 0.243 \\
\hline \multirow[t]{2}{*}{ Creatinine, mg/dL } & $\leq 1$ & 294 & 1.122 & $0.590-2.136$ & 0.725 \\
\hline & $>1$ & 222 & 0.445 & $0.152-1.301$ & 0.139 \\
\hline \multirow[t]{2}{*}{ AST, U/L } & $\leq 39$ & 262 & 0.836 & $0.370-1.889$ & 0.667 \\
\hline & $>39$ & 254 & 0.931 & $0.452-1.920$ & 0.847 \\
\hline \multirow[t]{2}{*}{$\mathrm{ALT}, \mathrm{U} / \mathrm{L}$} & $\leq 40$ & 255 & 0.790 & $0.390-1.597$ & 0.511 \\
\hline & $>40$ & 261 & 1.092 & $0.451-2.647$ & 0.845 \\
\hline
\end{tabular}

AFP alpha-fetoprotein, $P T$ prothrombin time, INR international normalized ratio, AST aspartate aminotransferase, $A L T$ alanine aminotransferase, $H R$ hazard ratio, $C I$ confidence interval; Values in bold, $P<0.05$ 


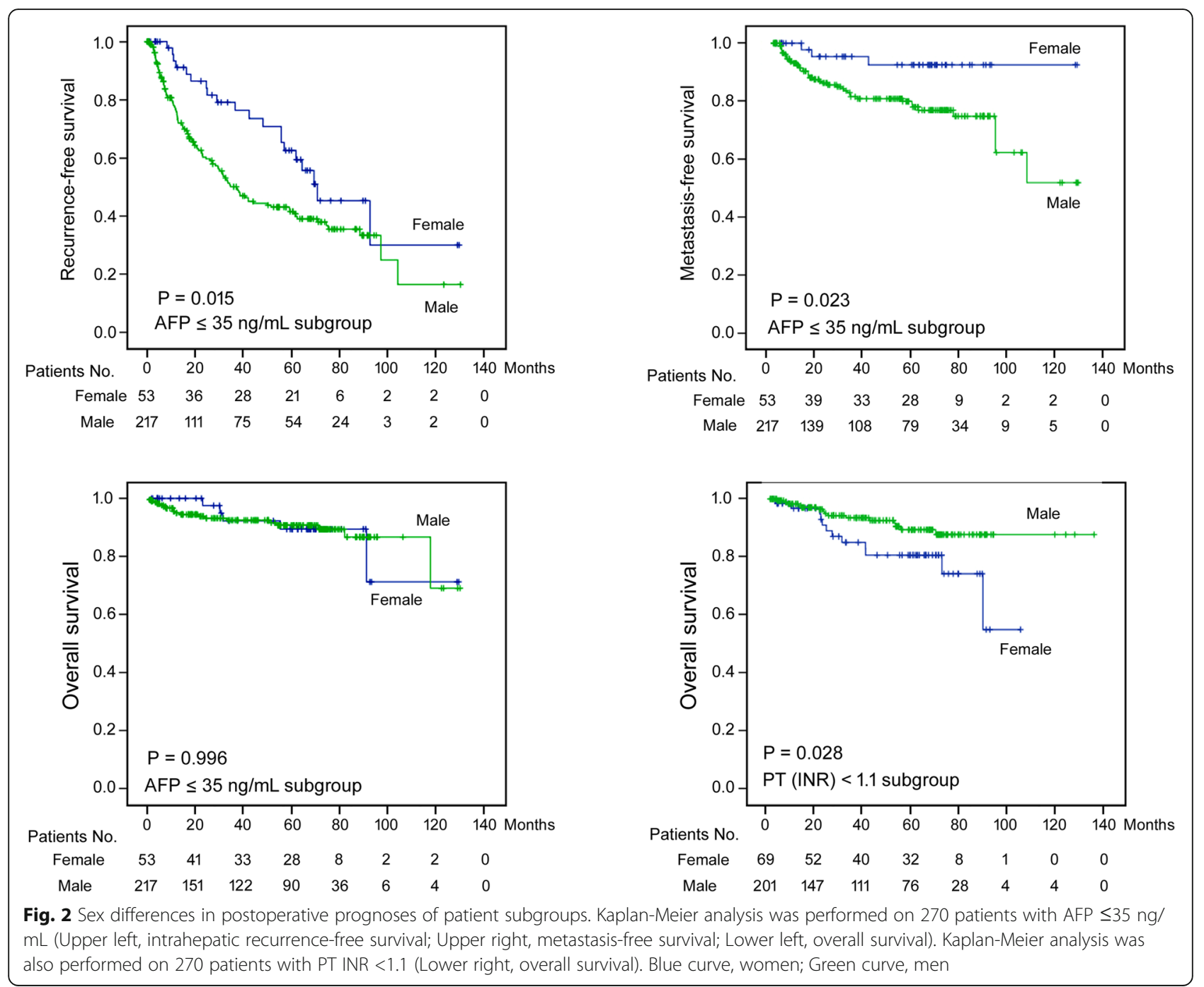

promotion, as well as estrogen/ estrogen receptor (ER) signaling mediated protection, of HCC, are driven by the Foxa1/Foxa2-dependent recruitment of ER- $\alpha$ and AR to target genes in a chemical-induced hepatocarcinogenesis mouse model. Foxa2 nucleotide polymorphisms may affect ER- $\alpha$ binding and correlate with the development of HCC in women [36]. Yang et al. demonstrated that estrogen reduces hepatocarcinogenesis through suppressing the alternative activation of macrophages (M2) via binding to ER- $\beta$, hence inhibiting JAK1-STAT6 signaling [15]. The correlation of lower risk and better survival of HCC with longer estrogen exposure in adult women (less parous, delayed menopause, hormone replacement therapy) has been proved in epidemiology studies in different populations [37-39]. Although animal models and epidemiology studies showed that sex hormones are determinants for the development and outcome of HCC, sex hormonetargeted therapies in HCC did not show a significant benefit over best supportive care $[40,41]$. The inconsistency in these results is attributed to inappropriate selection of patients with differential expression of receptors or variant receptors. Furthermore, ER- $\alpha 66$ (wild-type) and ER- $\alpha 36$ (splicing variant) were expressed inversely in non-tumor, non-cirrhotic to cirrhotic and cancerous stages [42], which enabled ER- $\alpha$ wild-type or variant transcripts in the tumor to be a better staging system for discriminating HCC prognosis than other scoring systems [43]. To understand the mechanism why female sex is associated with favorable postoperative outcome in HCC patients, it is essential to examine the estrogen and androgen levels before and after operation for all patients. It is possible that the sex hormone levels have a direct regulatory effect on HCC growth, or alternatively, host cells altered by long-term sex hormone stimulations could indirectly change the properties of cancer cell growth. In this retrospectively study, however, we were unable to measure the sex hormone levels. Besides, the average age of women with operable HCC in this study was 57.7 of age, indicating most of them were in 
Table 7 Cox proportional hazard analysis for clinical variables in relationship to overall survival in INR $<1.1$ subgroup $(n=270)$

\begin{tabular}{|c|c|c|c|}
\hline & HR & $95 \% \mathrm{Cl}$ & $P$ \\
\hline \multicolumn{4}{|l|}{ Univariate analysis } \\
\hline Age, per year & 1.003 & $0.977-1.029$ & 0.840 \\
\hline Gender, Male $=1$ & 0.438 & $0.205-0.936$ & 0.033 \\
\hline Anti-HCV, positive $=1$ & 1.277 & $0.540-3.021$ & 0.578 \\
\hline HBsAg, positive $=1$ & 0.501 & $0.232-1.081$ & 0.078 \\
\hline Liver cirrhosis, yes $=1$ & 1.112 & $0.522-2.366$ & 0.783 \\
\hline Microvascular invasion, yes $=1$ & 1.775 & $0.812-3.884$ & 0.151 \\
\hline Macrovascular invasion, yes $=1$ & 1.385 & $0.327-5.870$ & 0.659 \\
\hline Histology grade, per grade & 0.837 & $0.461-1.521$ & 0.560 \\
\hline Capsule, yes $=1$ & 0.974 & $0.411-2.309$ & 0.953 \\
\hline Tumor number, per number & 0.998 & $0.653-1.525$ & 0.994 \\
\hline Ascites yes $=1$ & 3.198 & $0.960-10.654$ & 0.058 \\
\hline Alcoholism yes $=1$ & 1.386 & $0.606-3.171$ & 0.440 \\
\hline Largest tumor size, per $\mathrm{cm}$ & 1.077 & $0.994-1.168$ & 0.071 \\
\hline AFP, per 1000 ng/mL & 1.002 & $0.998-1.006$ & 0.346 \\
\hline Albumin, per $\mathrm{g} / \mathrm{L}$ & 0.441 & $0.208-0.937$ & 0.033 \\
\hline Bilirubin, per mg/dL & 1.725 & $1.086-2.740$ & 0.021 \\
\hline PT, per sec & 0.695 & $0.374-1.293$ & 0.251 \\
\hline Creatinine, per mg/dL & 0.967 & $0.709-1.320$ & 0.834 \\
\hline AST, per U/L & 1.003 & $0.999-1.006$ & 0.193 \\
\hline ALT, per U/L & 1.000 & $0.995-1.005$ & 0.997 \\
\hline \multicolumn{4}{|l|}{ Multivariate analysis } \\
\hline Gender, Male $=1$ & 0.441 & $0.201-0.971$ & 0.042 \\
\hline Albumin, per $\mathrm{g} / \mathrm{dL}$ & 0.560 & $0.270-1.160$ & 0.119 \\
\hline Bilirubin, per mg/dL & 1.802 & $1.131-2.870$ & 0.013 \\
\hline
\end{tabular}

INR international normalized ratio, AFP alpha-fetoprotein, PT prothrombin time, AST aspartate aminotransferase, ALT alanine aminotransferase, $H R$ hazard ratio, $\mathrm{Cl}$ confidence interval; Values in bold, $P<0.05$

menopause and thus received less influence from estrogen, which could partly explain the similar postoperative prognosis between males and females.

\section{Conclusions}

In this retrospective cohort of patients with surgically resectable HCC, although no significant sex differences were found in OS, RFS and MFS, we found different prognosis between male and female patients, restricted to certain subgroups (in patients with lower AFP $\leq 35 \mathrm{ng} / \mathrm{mL}$, women showed better recurrence-free and metastasis-free survival; in patients with $\mathrm{PT}<1.1$, men showed better overall survival). The molecular mechanisms underlying this disparity may include interactions between sex hormone-related pathways and other growth-related signaling pathways at different stages of HCC.

\section{Additional files}

Additional file 1: Table S1. Sex specific 1-, 3-, and 5-year survival rates. The table lists 1-, 3-, and 5-year overall survival, metastasis-free survival, and recurrence-free survival rates in HCC patients of different sexes. (PDF 79 kb)

Additional file 2: Table S2. Clinicopathological factors associated with intrahepatic recurrence free survival in male and female HCC. The table lists univariate and multivariate analysis of clinicopathological factors associated with intrahepatic recurrence-free survival in HCC patients of different sexes. (PDF $91 \mathrm{~kb}$ )

Additional file 3: Table S3. Clinicopathological factors associated with distant metastasis free survival in male and female HCC. The table lists univariate and multivariate analysis of clinicopathological factors associated with distant metastasis free survival in HCC patients of different sexes, (PDF $91 \mathrm{~kb}$ )

Additional file 4: Table S4. Clinicopathological factors associated with overall survival in male and female HCC. The table lists univariate and multivariate analysis of clinicopathological factors associated with overall survival in HCC patients of different sexes. (PDF $91 \mathrm{~kb}$ )

\section{Abbreviations}

AFP: Alpha-fetoprotein; ALT: Alanine transaminase; AR: Androgen receptor; AST: Aspartate transaminase; BCLC: Barcelona Clinic Liver Cancer:

CLIP: Cancer of Liver Italian Program; CUPI: Chinese University Prognostic Index; ER: Estrogen receptor; GRETCH: Groupe d'Etude de Traitement du Carcinoma Hepatocellulaire; HBV: Hepatitis B virus; HCC: Hepatocellular carcinoma; HCV: Hepatitis C viru; HBsAg: HBV surface antigen;

INR: International normalized ratio; JIS: Japanese Integrated System; PT: Prothrombin time; TACE: Transarterial chemoembolization; TNM: TumorNode-Metastasis

\section{Acknowledgements}

We thank Ms. Chung-Yin Wu and Ms. Yu-Chiao Chuang for their diligent data collection.

\section{Funding}

This study was supported by Chang-Gung Memorial Hospital Research Program Grant (CORPG3G0971, CORPG3G1001). The funder had no role in study design, data collection, interpretation and analysis, or preparation of the manuscript.

\section{Availability of data and materials}

All data used for this study are available from the corresponding author upon reasonable request.

\section{Authors' contributions}

$M-W L$ and $C-T Y$, obtaining the funding, conducting the study, drafting the manuscript and critically revising the manuscript; C-T Y, C-L L, R-N C, and T-S $Y$, collecting and/or interpreting data; Y-D C, statistical analysis; T-L P, P-Y K, $\mathrm{K}-\mathrm{H} \mathrm{L}$, critical revising the manuscript for important intellectual content. All authors have read and approved the final version of the article.

\section{Ethics approval and consent to participate}

This study was approved by the Institutional Review Board, Chang Gung Memorial Hospital, Taiwan [The ethical approval reference number: 201700107B0]

\section{Consent for publication}

Not applicable.

\section{Competing interests}

The authors declare that they have no competing interests.

\section{Publisher's Note}

Springer Nature remains neutral with regard to jurisdictional claims in published maps and institutional affiliations. 


\section{Author details}

${ }^{1}$ Liver Research Center, Chang Gung Memorial Hospital, Taoyuan, Taiwan. ${ }^{2}$ Division of Pediatric Gastroenterology, Department of Pediatrics, Chang Gung Memorial Hospital, Taoyuan, Taiwan. ${ }^{3}$ Liver Research Unit, Chang Gung Memorial Hospital, Keelung, Taiwan. ${ }^{4}$ Department of Hepato-gastroenterology, Chang Gung Memorial Hospital, Taoyuan, Taiwan. ${ }^{5}$ Department of Surgery, Chang Gung Memorial Hospital, Taoyuan, Taiwan. ${ }^{6}$ School of Traditional Chinese Medicine, Chang Gung University, Taoyuan, Taiwan. ${ }^{7}$ Department of Biochemistry and Molecular Biology, Chang Gung University, Taoyuan, Taiwan. ${ }^{8}$ Department of Biochemistry, School of Medicine, Chang-Gung University, Taoyuan, Taiwan. ${ }^{9}$ Molecular Medicine Research Center, Chang Gung University, Taoyuan, Taiwan.

\section{Received: 15 September 2018 Accepted: 12 March 2019} Published online: 20 March 2019

\section{References}

1. Ferlay J, Soerjomataram I, Dikshit R, Eser S, Mathers C, Rebelo M, Parkin DM, Forman D, Bray F. Cancer incidence and mortality worldwide: Sources, methods and major patterns in GLOBOCAN 2012. Int J Cancer. 2015;136(5): E359-86. https://doi.org/10.1002/ijc.29210.

2. Knudsen ES, Gopal P, Singal AG. The changing landscape of hepatocellular carcinoma: etiology, genetics, and therapy. Am J Pathol. 2014;184(3):574-83. https://doi.org/10.1016/j.ajpath.2013.10.028.

3. El-Serag HB. Epidemiology of viral hepatitis and hepatocellular carcinoma. Gastroenterology. 2012;142(6):1264-1273.e1261. https://doi.org/10.1053/j. gastro.2011.12.061

4. McGlynn KA, London WT. The global epidemiology of hepatocellular carcinoma, present and future. Clin Liver Dis. 2011;15(2):223-43. https://doi. org/10.1016/j.cld.2011.03.006

5. Tanaka H, Imai Y, Hiramatsu N, Ito Y, Imanaka K, Oshita M, Hijioka T, Katayama K, Yabuuchi I, Yoshihara H. Declining incidence of hepatocellular carcinoma in Osaka, Japan, from 1990 to 2003. Ann Intern Med. 2008;148(11): 820-6. https://doi.org/10.7326/0003-4819-148-11-200806030-00004.

6. Davila JA, Morgan RO, Shaib Y, McGlynn KA, El-Serag HB. Hepatitis C infection and the increasing incidence of hepatocellular carcinoma: a population-based study. Gastroenterology. 2004;127(5):1372-80. https://doi. org/10.1053/j.gastro.2004.07.020.

7. Jemal A, Bray F, Center MM, Ferlay J, Ward E, Forman D. Global cancer statistics. CA Cancer J Clin. 2011;61(2):69-90. https://doi.org/10.3322/caac 20107.

8. Yuen MF, Hou JL, Chutaputti A. Hepatocellular carcinoma in the Asia pacific region. J Gastroenterol Hepatol. 2009;24(3):346-53. https://doi.org/10.1111/j. 1440-1746.2009.05784.x.

9. Farinati $F$, Sergio A, Giacomin A, Di Nolfo MA, Del Poggio P, Benvegnù L, Rapaccini G, Zoli M, Borzio F, Giannini EG. Is female sex a significant favorable prognostic factor in hepatocellular carcinoma? Eur J Gastroenterol Hepatol. 2009;21(10):1212-8. https://doi.org/10.1097/MEG.0b013e32831a86f8.

10. Hefaiedh R, Ennaifer R, Romdhane H, Ben Nejma H, Arfa N, Belhadj N, Gharbi L, Khalfallah T. Gender difference in patients with hepatocellular carcinoma. Tunis Med. 2013;91(8-9):505-8.

11. Jiang L, Shan J, Shen J, Wang Y, Yan P, Liu L, Zhao W, Xu Y, Zhu W, Su L, et al. Androgen/androgen receptor axis maintains and promotes cancer cell stemness through direct activation of Nanog transcription in hepatocellular carcinoma. Oncotarget. 2016;7(24):36814-28. https://doi.org/10.18632/ oncotarget.9192.

12. Naugler WE, Sakurai T, Kim S, Maeda S, Kim K, Elsharkawy AM, Karin M. Gender disparity in liver cancer due to sex differences in MyD88-dependent IL-6 production. Science. 2007;317(5834):121-4. https://doi.org/10.1126/ science.1140485

13. Nakagawa H, Maeda S, Yoshida H, Tateishi R, Masuzaki R, Ohki T, Hayakawa $Y$, Kinoshita $H$, Yamakado $M$, Kato $N$, et al. Serum IL-6 levels and the risk for hepatocarcinogenesis in chronic hepatitis C patients: an analysis based on gender differences. Int J Cancer. 2009;125(10):2264-9. https://doi.org/10. $1002 /$ ijc. 24720

14. Hou J, Xu J, Jiang R, Wang Y, Chen C, Deng L, Huang X, Wang X, Sun B. Estrogen-sensitive PTPRO expression represses hepatocellular carcinoma progression by control of STAT3. Hepatology. 2013:57:678-88. https://doi. org/10.1002/hep.25980.

15. Yang W, Lu Y, Xu Y, Xu L, Zheng W, Wu Y, Li L, Shen P. Estrogen represses hepatocellular carcinoma (HCC) growth via inhibiting alternative activation of tumor-associated macrophages (TAMs). J Biol Chem. 2012;287(48):401409. https://doi.org/10.1074/jbc.M112.348763.

16. Chen DS. Hepatocellular carcinoma in Taiwan. Hepatol Res. 2007;37(s2): S101-5.

17. Lee CM, Hung CH, Lu SN, Wang JH, Tung HD, Huang WS, Chen CL, Chen WJ, Changchien CS. Viral etiology of hepatocellular carcinoma and HCV genotypes in Taiwan. Intervirology. 2006;49(1-2):76-81.

18. Chang I-C, Huang S-F, Chen P-J, Chen C-L, Chen C-L, Wu C-C, Tsai C-C, Lee $\mathrm{P}-\mathrm{H}$, Chen M-F, Lee C-M, et al. The Hepatitis Viral Status in Patients With Hepatocellular Carcinoma: a Study of 3843 Patients From Taiwan Liver Cancer Network. Medicine. 2016;95(15):e3284. https://doi.org/10.1097/MD. 0000000000003284

19. Huang Y-T, Jen C-L, Yang H-I, Lee M-H, Su J, Lu S-N, Iloeje UH, Chen C-J. Lifetime risk and sex difference of hepatocellular carcinoma among patients with chronic hepatitis B and C. J Clin Oncol. 2011;29(27):3643-50. https://doi. org/10.1200/JCO.2011.36.2335.

20. Marrero JA, Fontana RJ, Barrat A, Askari F, Conjeevaram HS, Su GL, Lok AS. Prognosis of hepatocellular carcinoma: comparison of 7 staging systems in an American cohort. Hepatology. 2005;41(4):707-15. https://doi.org/10.1002/ hep.20636.

21. Grieco A, Pompili M, Caminiti G, Miele L, Covino M, Alfei B, Rapaccini GL, Gasbarrini G. Prognostic factors for survival in patients with earlyintermediate hepatocellular carcinoma undergoing non-surgical therapy: comparison of Okuda, CLIP, and BCLC staging systems in a single Italian centre. Gut. 2005;54(3):411-8. https://doi.org/10.1136/gut.2004.048124.

22. Cillo U, Vitale A, Grigoletto F, Farinati F, Brolese A, Zanus G, Neri D, Boccagni P, Srsen N, D'Amico F, et al. Prospective validation of the Barcelona Clinic Liver Cancer staging system. J Hepatol. 2006;44(4):723-31. https://doi.org/10. 1016/j.jhep.2005.12.015.

23. Gomaa Al, Hashim MS, Waked I. Comparing staging systems for predicting prognosis and survival in patients with hepatocellular carcinoma in Egypt. PLoS One. 2014;9(3):e90929. https://doi.org/10.1371/journal.pone.0090929.

24. Kitai S, Kudo M, Izumi N, Kaneko S, Ku Y, Kokudo N, Sakamoto M, Takayama T, Nakashima O, Kadoya M, et al. Validation of three staging systems for hepatocellular carcinoma (JIS Score, Biomarker-Combined JIS Score and BCLC System) in 4,649 cases from a Japanese nationwide survey. Dig Dis. 2014;32(6):717-24. https://doi.org/10.1159/000368008.

25. Bruix J, Reig M, Sherman M. Evidence-based diagnosis, staging, and treatment of patients with hepatocellular carcinoma. Gastroenterology. 2016; 150(4):835-53. https://doi.org/10.1053/j.gastro.2015.12.041.

26. Giannini EG, Farinati F, Ciccarese F, Pecorelli A, Rapaccini GL, Marco MD, Benvegnù L, Caturelli E, Zoli M, Borzio F, et al. Prognosis of untreated hepatocellular carcinoma. Hepatology. 2015;61(1):184-90. https://doi.org/10. 1002/hep.27443

27. Dohmen $\mathrm{K}$, Shigematsu $\mathrm{H}$, Irie $\mathrm{K}$, Ishibashi $\mathrm{H}$. Longer survival in female than male with hepatocellular carcinoma. J Gastroenterol Hepatol. 2003;18(3):26772. https://doi.org/10.1046/j.1440-1746.2003.02936.x.

28. Ladenheim MR, Kim NG, Nguyen P, Le A, Stefanick ML, Garcia G, Nguyen $\mathrm{MH}$. Sex differences in disease presentation, treatment and clinical outcomes of patients with hepatocellular carcinoma: a single-centre cohort study. BMJ Open Gastroenterology. 2016;3(1):e000107. https://doi.org/10. 1136/bmjgast-2016-000107.

29. Capuano G, Daniele B, Gaeta G, Gallo C, Perrone F. A new prognostic system for hepatocellular carcinoma: a retrospective study of 435 patients. Hepatology. 1998;28:751-5.

30. Chevret S, Trinchet J-C, Mathieu D, Rached AA, Beaugrand M, Chastang C. A new prognostic classification for predicting survival in patients with hepatocellular carcinoma. J Hepatol. 1999;31(1):133-41. https://doi.org/10. 1016/S0168-8278(99)80173-1

31. Leung TWT, Tang AMY, Zee B, Lau WY, Lai PBS, Leung KL, Lau JTF, Yu SCH, Johnson PJ. Construction of the Chinese University Prognostic Index for hepatocellular carcinoma and comparison with the TNM staging system, the Okuda staging system, and the Cancer of the Liver Italian Program staging system. Cancer. 2002;94(6):1760-9. https://doi.org/10.1002/cncr. 10384.

32. Burnett NP, Dunki-Jacobs EM, Callender GG, Anderson RJ, Scoggins CR, McMasters KM, Martin RC. Evaluation of alpha-fetoprotein staging system for hepatocellular carcinoma in noncirrhotic patients. Am Surg. 2013;79(7): 716-22.

33. Kinoshita A, Onoda H, Fushiya N, Koike K, Nishino H, Tajiri H. Staging systems for hepatocellular carcinoma: Current status and future 
perspectives. World J Hepatol. 2015;7(3):406-24. https://doi.org/10.4254/wjh. v7.3.406.

34. Subramaniam S, Kelley RK, Venook AP. A review of hepatocellular carcinoma (HCC) staging systems. Chinese Clinical Oncology. 2013;2(4):33.

35. Ma WL, Hsu CL, Yeh CC, Wu MH, Huang CK, Jeng LB, Hung YC, Lin TY, Yeh S, Chang C. Hepatic androgen receptor suppresses hepatocellular carcinoma metastasis through modulation of cell migration and anoikis. Hepatology. 2012;56(1):176-85. https://doi.org/10.1002/hep.25644.

36. Li Z, Tuteja G, Schug J, Kaestner Klaus H. Foxa1 and Foxa2 are essential for sexual dimorphism in liver cancer. Cell. 2012;148(1-2):72-83.

37. Yu MW, Chang HC, Chang SC, Liaw YF, Lin SM, Liu CJ, Lee SD, Lin CL, Chen PJ, Lin SC, et al. Role of reproductive factors in hepatocellular carcinoma: Impact on hepatitis B- and C-related risk. Hepatology. 2003;38(6):1393-400. https://doi.org/10.1016/j.hep.2003.09.041.

38. McGlynn KA, Hagberg K, Chen J, Braunlin M, Graubard BI, Suneja N, Jick S, Sahasrabuddhe W. Menopausal hormone therapy use and risk of primary liver cancer in the clinical practice research datalink. Int J Cancer. 2016; 138(9):2146-53. https://doi.org/10.1002/ijc.29960.

39. Hassan MM, Botrus G, Abdel-Wahab R, Wolff RA, Li D, Tweardy D, Phan AT, Hawk E, Javle M, Lee J-S, et al. Estrogen replacement reduces risk and increases survival times of women with hepatocellular carcinoma. Clin Gastroenterol Hepatol. 2017;15(11):1791-9. https://doi.org/10.1016/j.cgh.2017. 05.036 .

40. Nowak A, Findlay M, Culjak G, Stockler M. Tamoxifen for hepatocellular carcinoma. Cochrane Database Syst Rev. 2004;3:CD001024.

41. (GRETCH) GdFedTdCHe. Randomized trial of leuprorelin and flutamide in male patients with hepatocellular carcinoma treated with tamoxifen. Hepatology. 2004;40(6):1361-9. https://doi.org/10.1002/hep.20474.

42. Miceli V, Cocciadiferro L, Fregapane M, Zarcone M, Montalto G, Polito LM, Agostara B, Granata OM, Carruba G. Expression of wild-type and variant estrogen receptor alpha in liver carcinogenesis and tumor progression. OMICS. 2011;15(5):313. https://doi.org/10.1089/omi.2010.0108.

43. Villa E, Colantoni A, Camma C, Grottola A, Buttafoco P, Gelmini R, Ferretti I, Manenti F. Estrogen receptor classification for hepatocellular carcinoma: comparison with clinical staging systems. J Clin Oncol. 2003;21(3):441-6. https://doi.org/10.1200/JCO.2003.11.051.

Ready to submit your research? Choose BMC and benefit from:

- fast, convenient online submission

- thorough peer review by experienced researchers in your field

- rapid publication on acceptance

- support for research data, including large and complex data types

- gold Open Access which fosters wider collaboration and increased citations

- maximum visibility for your research: over $100 \mathrm{M}$ website views per year

At $\mathrm{BMC}$, research is always in progress.

Learn more biomedcentral.com/submissions 